\title{
Risk Factors for Poor Outcome in Patients with Severe Viral Pneumonia on Chest CT during the COVID-19 Outbreak: a Perspective from Iran
}

\author{
Amir H. Davarpanah ${ }^{1,2}$ (D) - Reyhaneh Asgari ${ }^{1}$ (D) Yashar Moharamzad $^{1}$ (D) $\cdot$ Arash Mahdavi $^{1}$ (D) - Alireza Abrishami ${ }^{1}$ (D) \\ Sayyedmojtaba Nekooghadam ${ }^{1}$ (D) $\cdot$ Ali Sabri $^{3}$ (D) Ehsan Zarei $^{1}$ (D) $\cdot$ Mehdi Khazaei $^{1}$ (D) $\cdot$ Morteza Sanei Taheri ${ }^{1,4}$ (D)
}

Accepted: 6 August 2020 / Published online: 19 August 2020

(C) Springer Nature Switzerland AG 2020

\begin{abstract}
We investigated significant predictors of poor in-hospital outcomes for patients admitted with viral pneumonia during the COVID-19 outbreak in Tehran, Iran. Between February 22 and March 22, 2020, patients who were admitted to three university hospitals during the COVID-19 outbreak in Tehran, Iran were included. Demographic, clinical, laboratory, and chest CT scan findings were gathered. Two radiologists evaluated the distribution and CT features of the lesions and also scored the extent of lung involvement as the sum of three zones in each lung. Of 228 included patients, 45 patients (19.7\%) required ICU admission and 34 patients $(14.9 \%)$ died. According to regression analysis, older age $(\mathrm{OR}=1.06 ; P<0.001)$, blood oxygen saturation $(\mathrm{SpO} 2)<88 \%(\mathrm{OR}=2.88 ; P=0.03)$, and higher chest $\mathrm{CT}$ total score $(\mathrm{OR}=1.10 ; P=0.03)$ were significant predictors for inhospital death. The same three variables were also recognized as significant predictors for invasive respiratory support: $\mathrm{SpO} 2<$ $88 \%(\mathrm{OR}=3.97, P=0.002)$, older age $(\mathrm{OR}=1.05, P<0.001)$, and higher $\mathrm{CT}$ total score $(\mathrm{OR}=1.13, P=0.008)$. Potential predictors of invasive respiratory support and in-hospital death in patients with viral pneumonia were older age, $\mathrm{SpO} 2<88 \%$, and higher chest CT score.
\end{abstract}

Keywords Tomography, X-ray computed · Pneumonia $\cdot$ COVID-19 $\cdot$ Prognosis $\cdot$ Patient outcome assessment

\section{Introduction}

Since late December 2019 when a new coronavirus was isolated from a group of patients diagnosed with pneumonia in
China, the world has observed rapid expansion of this novel contagious virus called SARS-CoV-2 (severe acute respiratory syndrome coronavirus 2 ). The disease caused by this novel virus is called coronavirus disease (COVID-19), and its
Amir H. Davarpanah

amir.davarpanah@emory.edu

Morteza Sanei Taheri

saneim@gmail.com; m_sanei@sbmu.ac.ir

Reyhaneh Asgari

reyhaneasgari@yahoo.com

Yashar Moharamzad

yasharpop@hotmil.com

Arash Mahdavi

arashmahdavi@gmail.com

Alireza Abrishami

abr.alireza@yahoo.com

Sayyedmojtaba Nekooghadam

samonemot@sbmu.ac.ir
Ali Sabri

sabri.ali@gmail.com

Ehsan Zarei

md.ehsanzarei@gmail.com

Mehdi Khazaei

medc2018@gmail.com

School of Medicine, Shahid Beheshti University of Medical Sciences, Tehran, Iran

2 Department of Radiology and Imaging Sciences, Emory University Hospital, School of Medicine, Emory University, Atlanta, GA 30322, USA

3 Niagara Health, McMaster University, Hamilton, Ontario, Canada

4 Department of Radiology, Shohada Hospital, Tajrish Sq, Tehran 1445613131, Iran 
clinical presentation spectrum ranges from asymptomatic infection to severe pneumonia which necessitates hospital admission and its severe complication, acute respiratory distress syndrome (ARDS), which warrants intensive care unit (ICU) admission, intubation, and pulmonary support care $[1,2]$.

Meanwhile that our knowledge about diagnosis, clinical course, and outcome of COVID-19 is improving, a major challenge for healthcare systems is the overwhelming number of patients admitted due to upper respiratory tract infection (URI) symptoms. A major challenge for clinicians, amid shortage of ICU beds and ventilators current centers worldwide are experiencing [3], is to determine the prognostic factors that significantly influence the clinical outcome of the patients.

Some clinical and laboratory factors have been noted to be associated with severe illness and mortality in COVID-19. Older age ( $\geq 65$ years) was confirmed in two studies as a significant risk factor for development of ARDS and death $[4,5]$. Case fatality rate was noted to be higher in patients with comorbidities, in particular cardiovascular diseases, diabetes, and chronic respiratory disease [6]. In a previous study including 191 patients in China to determine risk factors for fatal COVID19 during hospitalization reported older age, serum d-dimer > $1 \mathrm{mcg} / \mathrm{mL}$ on admission, and higher Sequential Organ Failure Assessment (SOFA) score as significant predictors for inhospital death [4]. In this study, even though consolidation $(74 \%$ vs. $53 \%)$ and ground glass opacity (GGO) $(81 \%$ vs. $67 \%$ ) on chest $\mathrm{CT}$ scans were significantly more prevalent in non-survivors compared with those who survived, CT features were not entered into the multivariable logistic regression analysis. The other study which explored the risk factors for progression from ARDS to death in 201 patients did not investigate the severity of pneumonia on chest CT, albeit $95 \%$ of the patients had bilateral infiltrates on chest imaging [5].

According to the consensus statement made by RSNA (Radiologic Society of North America) and STR (Society of thoracic Radiology), chest CT scan should not be used for routine screening of suspected COVID-19 pneumonia patients [7]. Chest CT scan should be reserved for hospitalized, symptomatic patients with specific clinical indications for imaging when complications are suspected. In addition to typical findings that may be characteristic for COVID-19 pneumonia (i.e., peripheral or bilateral GGO, crazy paving, reverse halo sign) [7], the course of progression or recovery of the infection could be monitored by chest CT scan $[8,9]$. Increase in consolidative opacities and GGO as well as interstitial septal thickening on chest CT scan has been described in patients with worsening pneumonia $[10,11]$. Chest $\mathrm{CT}$ scan has also been reported as a sensitive method when correlating with reverse-transcription polymerase chain reaction (RT-PCR) in making the diagnosis [12]. At the peak of COVID-19 outbreak, Iranian health authorities broadened the official definition of infection to include patients with typical findings at CT even with initial negative PCR results $[13,14]$. This decision resulted in higher number of suspected COVID-19 patients and surge of suspected pneumonia patients requiring hospital admission. Meanwhile, as global coronavirus cases surge past 2 million, there is a pressing need to identify prognostic factors in patients with suspected COVID-19 pneumonia, given current shortage of medical supplies, healthcare staff, and ICU beds across the world, specifically in resource-constrained environments. Therefore, we intend to report prognostic factors for ICU admission and mortality in our patient cohort and investigate demographic, clinical, laboratory, and radiologic features that could be helpful in initial management and risk stratification of patients. In particular, to overcome the shortage of two previous studies where the role of imaging findings was underestimated $[4,5]$, we assessed the role of chest CT score as potential predictor for in-hospital death. In our opinion, the results of this study would be helpful for clinicians and radiologists who provide care for patients with suspected viral pneumonia and enable them to use the suggested prognostic factors for better management of at-risk patients.

\section{Materials and Methods}

\section{Cohort}

In this retrospective study, we included adult patients who were admitted to three major university hospitals affiliated to Shahid Beheshti University of Medical Sciences, Tehran, Iran between February 22 and March 22, 2020 with suspected viral pneumonia. The admission criteria for viral pneumonia were according to guidelines published by the Iranian Ministry of Health for management of patients with suspected COVID-19 pneumonia and included a combination of clinical, laboratory, and radiologic information [15]. In summary, the COVID-19 outbreak in Iran was declared in mid-February 2020 and since then, the health authorities have released and updated mandatory guidelines describing the diagnostic criteria for COVID19, outpatient management, hospitalization indications, and pharmacotherapy for healthcare staff in designated hospitals throughout Iran (especially emergency medicine, internal medicine, infectious disease specialists, and general practitioners) in an effort to provide a standard and uniform management algorithm for patients with suspected COVID-19 pneumonia. In the early days of the outbreak in Iran, shortage of RT-PCR test kits hampered appropriate disease screening. Therefore, clinical findings including exposure history, fever, and respiratory symptoms (in particular dyspnea and decreased blood oxygen saturation ( $\mathrm{SpO} 2)$ ), laboratory findings (C-reactive protein, ESR, and lymphopenia), and chest CT scan were frequently used by healthcare facilities to determine the need for SARS-CoV-2 RT-PCR testing and further admission. According to the guidelines, patients who present with respiratory symptoms in case of presence of dyspnea, 
Fig. 1 Flowchart of the patients with viral pneumonia during the COVID-19 outbreak included in the study
793 patients with respiratory symptoms who presented to emergency rooms and were examined by chest chest $\mathrm{CT}$

361 ptients had chest CT findings suggestive for viral pneumonia
133 patients were excluded (103 patients were not admittted and 30 patients had missing data) respiratory rate over 30 per minute, or $\mathrm{SpO} 2<93 \%$ require referral to the designated hospitals. Chest CT scan is accessible in the studied hospitals and was performed for all patients. Exclusion criteria were clinical and CT findings inconsistent with viral pneumonia (including bacterial pneumonia, exacerbation of chronic obstructive pulmonary disease, reactive airway disease, congestive heart failure, etc.) and patients with incomplete follow-up.

\section{Data Collection}

First, a data gathering form was designed after the literature review. In this checklist, demographic (age and gender), clinical, laboratory, and chest CT scan findings of the patients were entered. Five researchers (two clinicians and three radiologists) reviewed the medical records of the patients who met the inclusion criteria during the study period and extracted the required data. The clinical variables included vital signs (fever (oral temperature $\geq 38{ }^{\circ} \mathrm{C}$ ), tachypnea (respiratory rate $>20$ breaths per minute), tachycardia (pulse rate $>100$ beats per minute), SpO2, dominant symptoms upon admission, and pre-existing comorbidities (ischemic heart disease, diabetes mellitus, chronic respiratory disease, etc). Laboratory data included CBC (complete blood count), liver function tests (ALT and AST), renal function tests (BUN and creatinine), venous blood gas (VBG) analysis, electrolytes, quantitative CRP, ESR (erythrocyte sedimentation rate), and LDH (lactate dehydrogenase). Leukocytosis was defined as elevated white blood

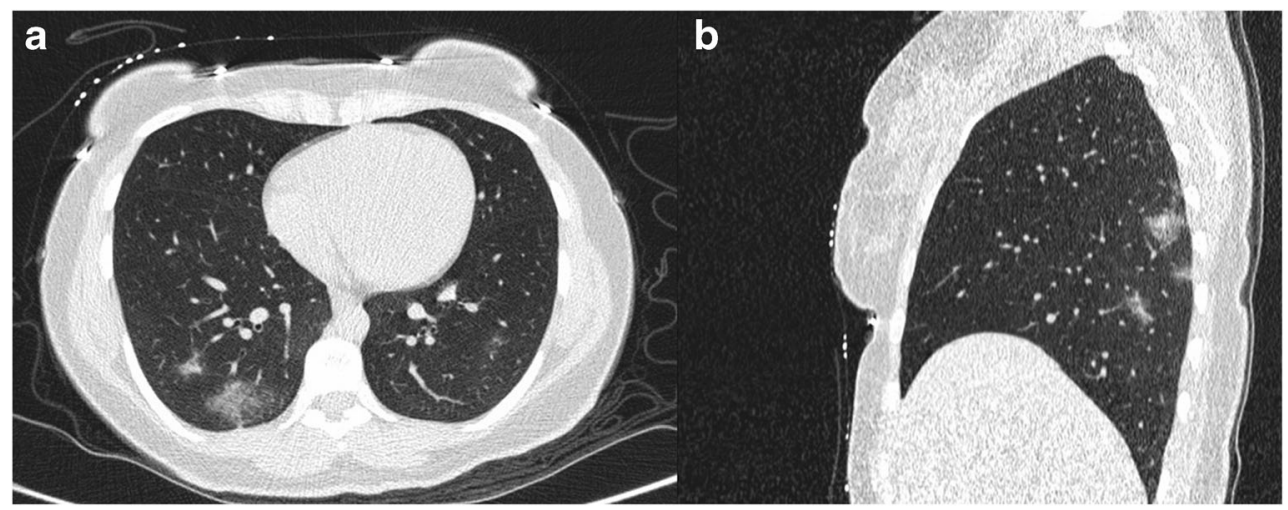

Fig. 2 A 30-year-old female presented with fever and cough. Her initial blood oxygen saturation was $96 \%$ on room air. Axial (A) and sagittal (B) reconstructions of low dose spiral CT scan of the chest revealed patchy ground glass opacities most prominently in bilateral lower lobes with total CT score of 5. The patient was admitted to the ward and discharged after six days with good general conditions 
cell (WBC) count greater than 11,000 per $\mathrm{mm}^{3}$, and lymphopenia was defined as lymphocyte count less than 1500 per $\mathrm{mm}^{3}$. Abnormal creatinine was defined as serum creatinine greater than $1.2 \mathrm{mg} / \mathrm{dl}$. Abnormal liver function test values used are as follows: AST above $40 \mathrm{IU} / \mathrm{mL}$, ALT values above $56 \mathrm{IU} / \mathrm{mL}$, ALP above $140 \mathrm{IU} / \mathrm{L}$. Abnormal PaCO2 on venous blood gas (VBG) was defined as values above $45 \mathrm{mmHg}$. Results of RT-PCR testing was included if available.

\section{Image Analysis}

Two board-certified radiologists (AM and AA, with 6 and 8 years of experience in chest radiology, respectively) interpreted chest CT scans using consensus agreement. Findings suggestive of viral pneumonia were identified and recorded according to published literature $[8,16]$ including patchy ground glass opacities and consolidation with peripheral and multilobar involvement, opacities with rounded morphology, linear opacities, crazy paving appearance, and reverse halo sign. Then, the extent of involvement on CT images was also assessed by the two radiologists using consensus agreement according to the method by Zhou et al. [17]. In brief, each lung was divided into three zones in craniocaudal direction. The degree of involvement in each lung zone was scored as follows: a score of 0 denoted no involvement; $1,<$ $25 \%$ involvement; $2,25 \%$ to less than $50 \%$ involvement; 3 , $50 \%$ to less than $75 \%$ involvement; and $4, \geq 75 \%$ involvement. Scores were recorded and summed for each lung with maximum possible score of 24 .

\section{Outcomes}

Two outcomes (in-hospital death and requirement for ICU admission) were defined for the patients. Definitive outcomes were recorded as of April 15, 2020. ICU admission was determined by the treating physician due to need for invasive respiratory support including orotracheal intubation and/or mechanical ventilation.

\section{Statistical Analysis}

Statistical analysis was conducted using SAS Version 9.4. For numeric covariates, the mean and SD were calculated and presented. Frequency and its percentage were shown for categorical variables. To compare differences between patients required and did not require ICU admission as well as survivors and non-survivors, we used one-way analysis of variance (ANOVA) test and Kruskal-Wallis test for numerical covariates and chi-square test or Fisher's exact test for categorical covariates where appropriate. To explore the risk factors associated with ICU admission and in-hospital death, univariable and multivariable logistic regression models were used. We excluded variables from the univariable analysis if their between-group differences were not significant, if the number of events was too small to calculate odds ratios, or if they had collinearity with other included factors. The significance level was set at 0.05 .

\section{Ethical Considerations}

The study protocol was approved by the Ethics Committee of Research Deputy of Shahid Beheshti University of Medical Sciences, Tehran, Iran. The study was performed considering the ethical principles outlined in the Declaration of Helsinki.

\section{Results}

A total of 228 patients (148 (52.4\%) males and $134(47.6 \%)$ females) with a mean $( \pm \mathrm{SD})$ age of 56.1 years $( \pm 15.9)$ were included (Figs. 1, 2, and 3). Positive RT-PCR for SARS-CoV2 was available for a subset of studied patients $(n=94$, $41.2 \%)$. The remainder of patients $(n=134,58.8 \%)$ were treated according to clinicolaboratory and CT criteria of viral infection. Forty-five patients (19.7\%) required ICU admission, and 34 patients $(14.9 \%)$ died. Of the 34 patients who died, 33 patients had been admitted to ICU (97.06\%), and only

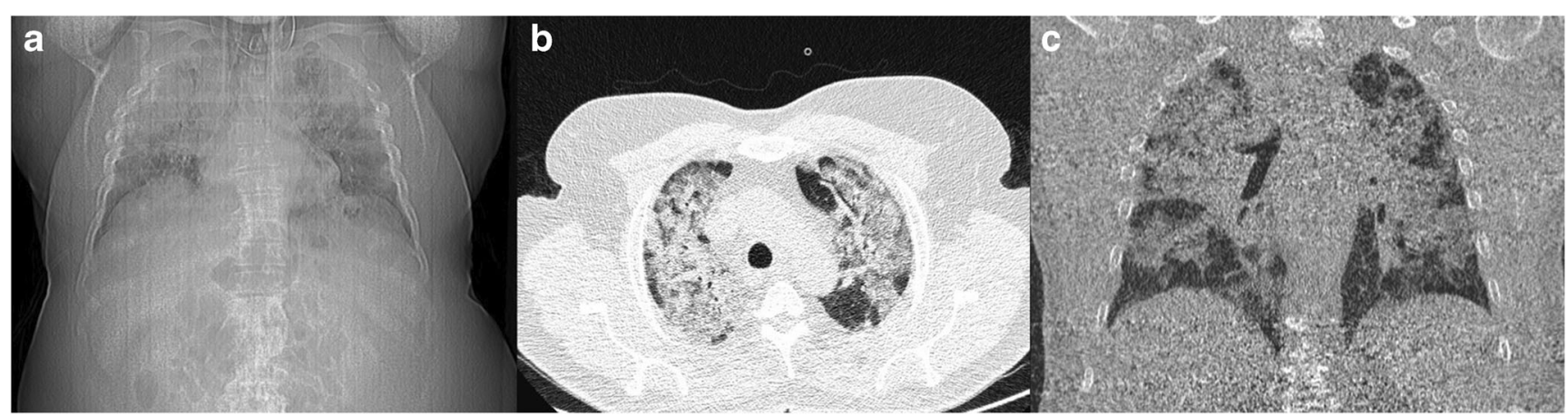

Fig. 3 A 65-year-old male presented with fever and dyspnea. His blood oxygen saturation was $88 \%$ on room air with respiratory rate of 40 per minute. Scout view demonstrated severe bilateral air-space consolidation (A). CT scan also revealed widespread patchy air-space consolidation in bilateral lungs, more prominent in the upper lung regions, with calculated total CT score of 19 (B and C). Subsequently, the patient was admitted to the intensive care unit 
one patient $(2.94 \%)$ had not been admitted to ICU $(P<0.001)$. Mean $( \pm \mathrm{SD})$ age of patients who required ICU admission $(65$ \pm 15.8 years) was significantly higher than those who were not admitted to ICU ( $53.9 \pm 15.1$ years); $P<0.001$. Likewise, patients who died during hospitalization were older (67.5 \pm 14.6 years $)$ compared with survivors $(54 \pm 15.2$ years $)$; $P<0.001$. The most common clinical symptoms at presentation were cough and shortness of breath. Hypertension followed by diabetes mellitus (DM) were the two most common comorbidities. Between patients with and without RT-PCR testing results, there was no significant difference between age $(53.6 \pm 15.0$ vs. $57.7 \pm 16.3$ years; $P=0.068)$, rate of $\mathrm{SpO} 2$ below $88 \%$ (66/154 [42.9\%] vs. 88/154 [57.1\%]; $P=$ $0.071)$, total CT score $(9.7 \pm 5.4$ vs. $8.4 \pm 4.3 ; P=0.062)$, ICU admission (23/45 [51.1\%] vs. $22 / 45$ [48.9\%]; $P=0.176)$, and in-hospital death $(15 / 34$ [44.1\%] vs. 19/34 [55.9\%]; $P=0.71)$.

Table 1 presents clinical characteristics (vital signs, presenting symptoms, and pre-existing comorbidities) in the whole sample and univariate analyses between patients who were admitted to ICU and those who were not as well as survivors and non-survivors. As observed, except coughing, tachycardia, $\mathrm{SpO} 2$ on admission, and chronic renal failure, no statistically significant differences were detected regarding other variables between ICU admitted and non-admitted as well as non-survivors and survivors.

Leukocytosis was more prevalent in ICU admitted patients and also among non-survivors. On the other hand, no difference between survivors and non-survivors was seen regarding lymphopenia. Renal function tests (serum blood urea nitrogen and creatinine) showed higher proportion of abnormal values in those who were admitted to ICU as well as in non-survivors. Serum CRP level was higher in the non-survivors group. However, serum ESR level did not differ between the groups. Table 2 presents biochemical laboratory tests upon admission to the hospitals.

On chest CT examination, the three most common abnormalities were GGO (67.1\%), linear opacities (33.3\%), and consolidation (18.9\%). Pleural effusion and bronchial wall thickening were more common in non-survivors compared with survivors based on univariate analyses. Table 3 summarizes abnormal chest $\mathrm{CT}$ scan findings.

Mean $( \pm \mathrm{SD})$ total CT score was $8.96( \pm 4.83)$ in the whole sample. The total CT score was significantly higher in patients admitted to ICU $(11.9 \pm 5.8)$ than in those who did not require ICU admission ( $8.2 \pm 4.3) ; P<0.001$. Similarly, the score was significantly higher in patients who died $(12.2 \pm 5.9)$ than in survivors $(8.4 \pm 4.4): P<0.001$.

\section{Multivariable Logistic Regression Analysis}

In order to determine significant risk factors for in-hospital death, age, $\mathrm{SpO} 2(<88 \%$ vs. $>88 \%)$, total CT score, leukocytosis, blood pH level, and serum creatinine ( $<1.2$ vs. $>1.2)$ were entered into the analysis. The results showed that age, total $\mathrm{CT}$ score, and $\mathrm{SpO} 2$ were significant predictors for ICU admission and mortality (Table 4).

\section{Discussion}

Since the report of the COVID-19 outbreak in December 2019 in China and rapid spread of this infection in more than 200 countries which resulted in the COVID-19 pandemic declaration by the WHO, a major challenge for healthcare staff is the surge in the number of patients suspected to have COVID-19 pneumonia which has overwhelmed hospitals. The rapid growth of critically ill patients and limited health resources, especially ICU beds and ventilators, has necessitated effective triage strategies for identifying patients at greatest risk for worse outcome to achieve effective resource allocation for both outpatient and inpatients [18]. Several triage strategies have been proposed so far for patients with acute upper respiratory symptoms during the COVID-19 outbreak $[19,20]$ focusing more on outpatient management. On the other hand, limited data is currently available on risk stratification of admitted patients with viral pneumonia including COVID-19 pneumonia as many hospitals around the world are struggling with ICU bed shortage [21].

In this study, we decided to determine significant prognostic factors in a cohort of admitted patients whose clinical presentation, lab, and chest CT findings were suggestive of viral pneumonia. Similar to other countries, diagnostic kits were not accessible in all hospitals in Iran, and therefore, doctors faced challenges to manage and triage suspected patients appropriately given the knowledge of the ongoing COVID-19 epidemic in Iran. Our results indicated that older age, $\mathrm{SpO} 2<$ $88 \%$, and higher chest CT score were significant predictors for both invasive repertory support or in-hospital death during the current outbreak in our university medical centers. Older age has been confirmed in two previous similar studies as independent prognostic factor for death in confirmed COVID-19 pneumonia patients $[4,5]$. In a preliminary report of 121 patients admitted to ICU in the USA, $80 \%$ of those who died were older than 65 years [22]. Comorbidities are more common in older patients, especially cardiovascular diseases and chronic respiratory conditions. These comorbidities may contribute in severe pneumonia and development of ARDS in older patients [23]. However, none of the studied comorbidities in the sample had significant role, and older age was recognized as independent risk factor for in-hospital death.

Another identified prognostic factor of poor outcome was $\mathrm{SpO} 2<88 \%$ on admission of the patients. Since $\mathrm{SpO} 2$ measurement is a non-invasive method to evaluate blood oxygenation, this item has been incorporated in some guidelines, including guidelines published by the Iranian Ministry of Health, to identify high-risk patients who require hospital 


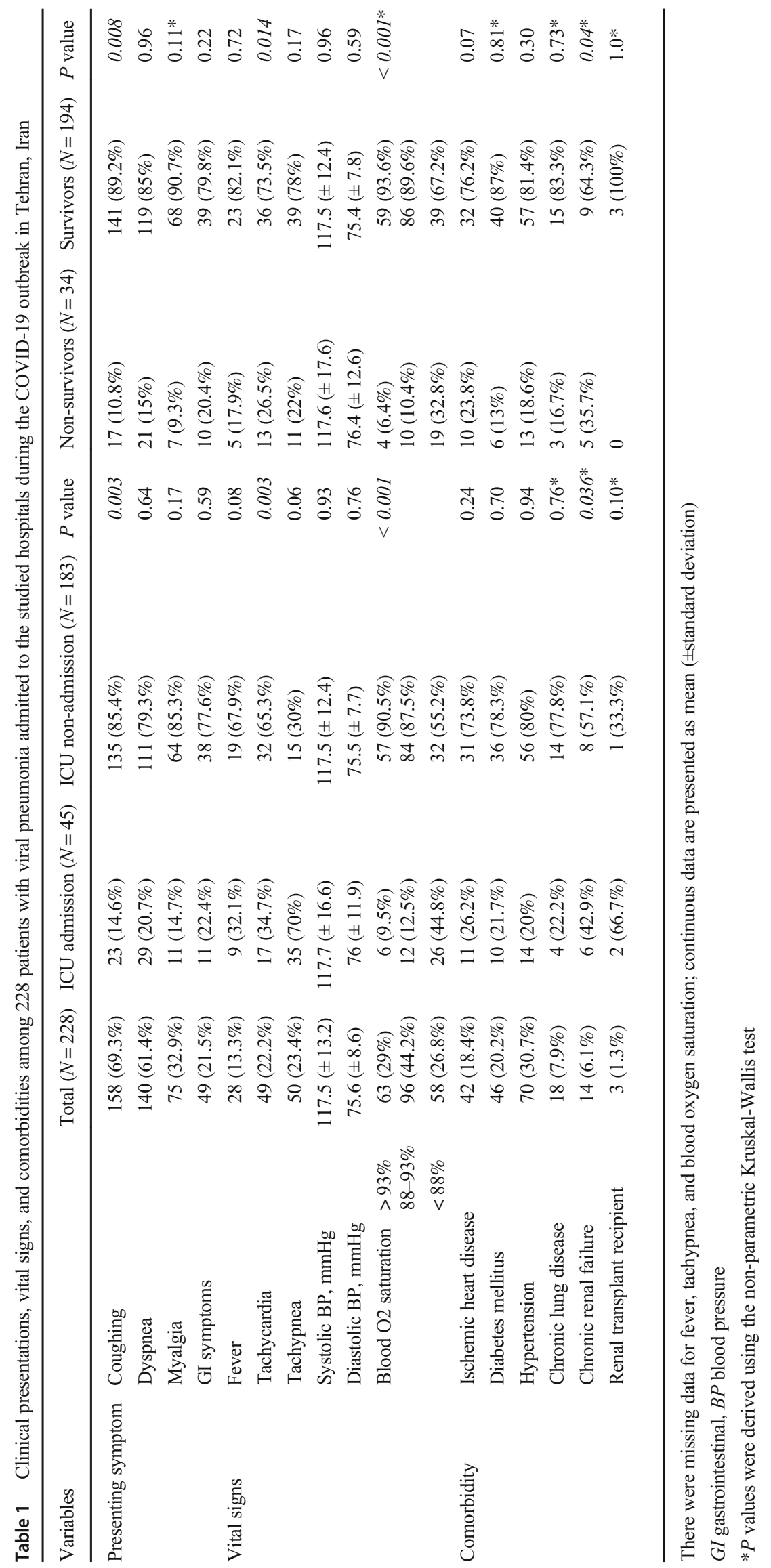




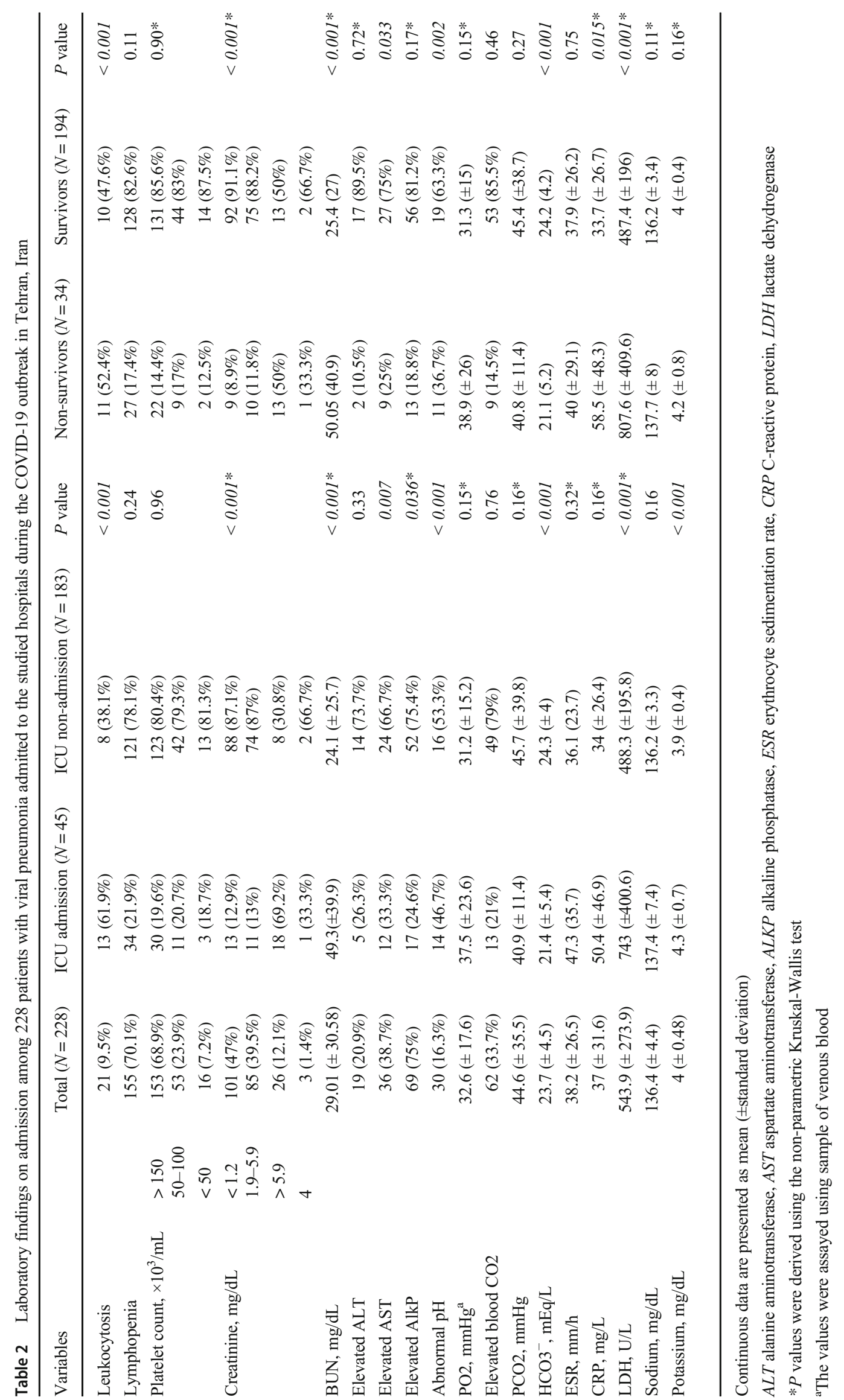




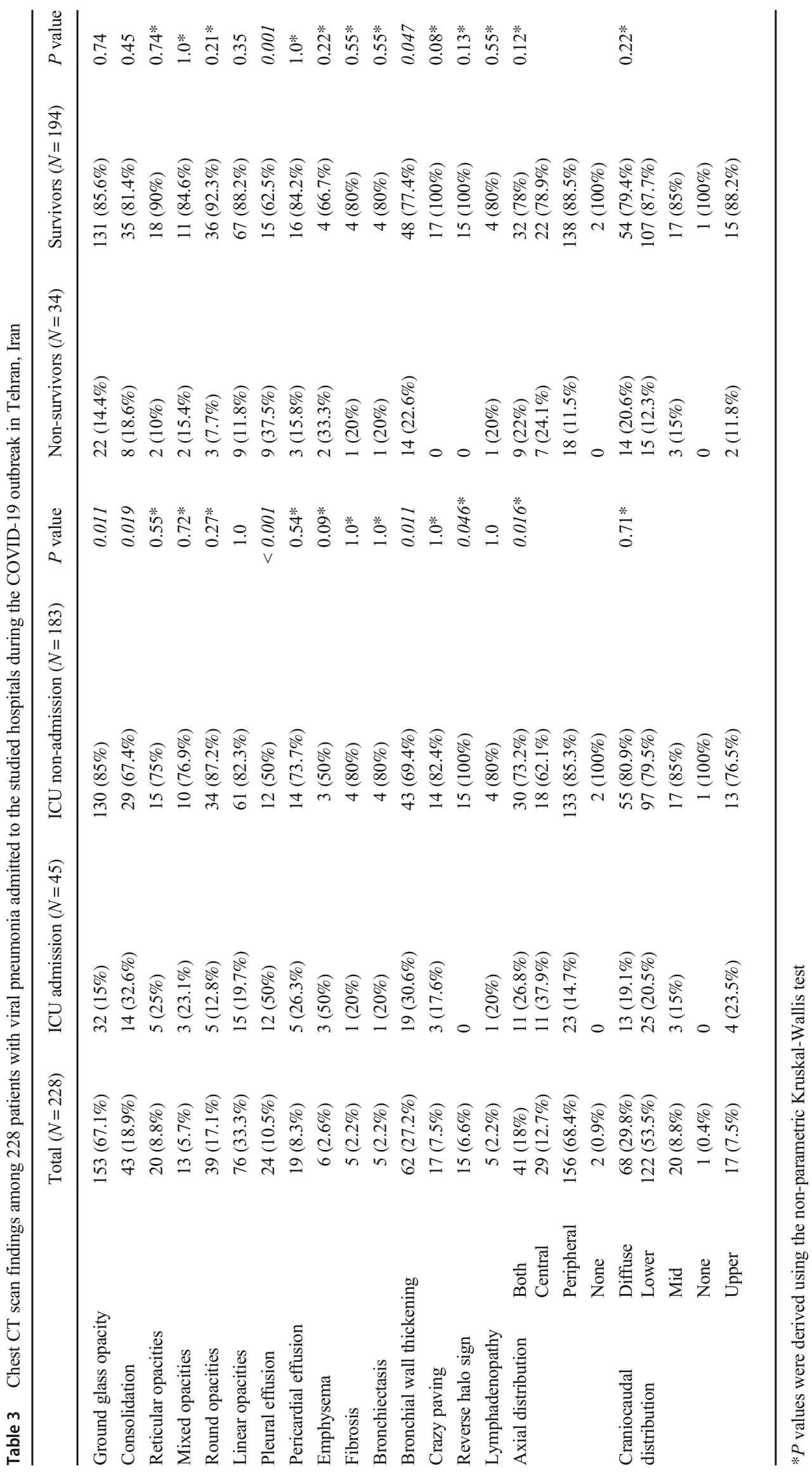


Table 4 Results of multivariable logistic regression model to determine significant predictors for ICU admission and in-hospital death among 228 patients with viral pneumonia admitted to the studied hospitals during the
COVID-19 outbreak in Tehran, Iran. $\mathrm{SE}=$ standard error; $\mathrm{OR}=$ odds ratio; $\mathrm{CI}=$ confidence interval

\begin{tabular}{|c|c|c|c|c|c|c|}
\hline Outcome & Variables & Beta (estimate) & SE & $P$ value & Adjusted OR & $95 \% \mathrm{CI}$ \\
\hline \multirow[t]{4}{*}{ In-hospital death } & Age (per 1 year increase) & 0.06 & 0.01 & $<0.001$ & 1.06 & 1.03 to 1.10 \\
\hline & Total CT score (per 1 score increase) & 0.10 & 0.05 & 0.03 & 1.10 & 1.00 to 1.22 \\
\hline & Blood oxygen saturation $<88 \%$ & -0.52 & 0.24 & 0.03 & 2.88 & 1.09 to 7.62 \\
\hline & Intercept & -6.77 & 1.41 & $<0.001$ & & \\
\hline \multirow[t]{4}{*}{ ICU admission } & Age (per 1 year increase) & 0.05 & 0.01 & $<0.001$ & 1.05 & 1.02 to 1.09 \\
\hline & Total CT score (per 1 score increase) & 0.12 & 0.04 & 0.008 & 1.13 & 1.02 to 1.24 \\
\hline & Blood oxygen saturation $<88 \%$ & -0.68 & 0.22 & 0.002 & 3.97 & 1.61 to 9.74 \\
\hline & Intercept & -5.83 & 1.26 & $<0.001$ & & \\
\hline
\end{tabular}

referral or admission [19]. Additionally, $\mathrm{SpO} 2$ less than $90 \%$ with shortness of breath and respiratory rate more than 30 per minute have been suggested as evidence of severe COVID-19 pneumonia in febrile patients [24]. Low blood oxygen saturation has also been used to identify severe COVID-19 pneumonia in admitted patients [25]. $\mathrm{SpO} 2$ has previously been shown to be a valuable prognostic tool in communityacquired pneumonia with good specificity for poor outcome [26], and based on our findings, the odds of ICU admission and mortality were respectively four times and three times higher when $\mathrm{SpO} 2$ was lower than $88 \%$ on admission.

As described previously, routine chest $\mathrm{CT}$ scans are not recommended for diagnosis of COVID-19. In the early phase of the COVID-19 outbreak in Iran, since access to RT-PCR was not available in many hospitals, chest CT scan was used temporarily to identify patterns consistent with COVID-19 pneumonia to screen infected individuals. Therefore, we were able to integrate imaging data with clinical and laboratory findings to identify the most significant prognostic factors for poor outcome in our cohort. The total CT score was also identified as significant prognostic factor for both outcomes.

Ground glass opacity and consolidation were more common in patients who required ICU admission. These findings demonstrate that chest $\mathrm{CT}$ scan could be useful risk stratification tool for admitted patients, and by applying a simple method for scoring the abnormal findings in each zone, radiologists could provide critical information regarding at-risk patients who are more likely to require ICU admission. Ground glass opacity has been reported to be a very common finding in COVID-19 pneumonia as $100 \%$ of patients whose diagnosis was confirmed by RT-PCR had this finding in a study of 58 patients in Italy [27]. In the mentioned study [27], the authors reported that none of the CT features (i.e., ground glass opacity, bilateral distribution of pneumonia, involvement in more than two lobes, consolidation, and lymphadenopathy) were significantly different between those with confirmed COVID-19 who required admission vs. those who were discharged for home isolation. In a study on 73 COVID-19 patients which included 25 patients with severe or critical condition, a CT scoring system was used where higher scores had significant relationship with more severe disease [28]. Although the scoring system used by this study assigns different scores from the scoring system we used here, similar to what we observed here, CT scores were significantly higher in critical patients. Pleural effusion and bronchial wall thickening were two important abnormalities more frequently observed in non-survivors. These are in agreement with $\mathrm{Li}$ et al.'s study [28].

\section{Limitations}

First, given temporary test kit shortages in Iran, RT-PCR was not performed for all patients in our cohort. All patients included in the study had chest CT pattern consistent with typical findings of viral pneumonia and clinical findings as well as laboratory findings were suspicious for viral pneumonia which are concordant with the Fleischner Society Consensus Statement on the use of chest $\mathrm{CT}$ in patient management during the COVID-19 pandemic in resource-constrained environments [29]. It is also known that the findings on chest imaging in COVID-19 are not specific, and overlap with other infections, including influenza among others, limiting specificity of CT. Nonetheless, it is known that acute respiratory distress syndrome (ARDS) can be induced by a variety of viral diseases, and triaging infected individuals regardless of etiology is of vital importance specifically in the midst of the current global pandemic. Second, other specific markers of worse outcome such as interleukin levels were not measured routinely and were not available for data collection. For a very limited number of patients, serum d-dimer had been measured which were within normal range. Therefore, we decided not to include this laboratory marker although a previous study suggested that d-dimer was a significant risk factor for death. It should be noted that other inflammation markers had been assayed. But, 
neither ESR nor quantitative CRP was recognized as significant risk factor for mortality.

\section{Conclusion}

Since many hospitals may have difficulties with limited advanced respiratory care supplies and ICU beds during the COVID-19 outbreak and considering the importance of triaging for severe viral pneumonia patients, it is recommended that older patients with $\mathrm{SpO} 2$ less than $88 \%$ and multilobar involvement on chest CT scan on admission (reflected as higher total CT score) be prioritized for invasive respiratory support measures. In our opinion, these findings could help clinicians and radiologists for better decision-making for admitted patients with viral pneumonia in time of COVID-19 pandemic.

Author Contributions Conceptualization: MST, AHD, AS, AM. Methodology: All authors. Project administration: YM and MST. Data gathering: RA, AM, AA, SN, MKh, SN. Supervision: MST, SN, AHD. Writing of original draft: YM, AHD, MK. Review and Editing: all authors.

Funding Information No fund was received to conduct this study.

\section{Compliance with Ethical Standards}

Conflict of Interest The authors declare that there is no conflict of interest.

Ethical Approval The study was approved by the Ethics Committee of Research Deputy, Shahid Beheshti University of Medical sciences, Tehran, Iran.

Informed Consent Since the required data were obtained from medical records of patients already admitted to the hospital and informed consent is obtained upon admission for diagnostic and therapeutic purposes, informed consent for this study was waived.

\section{References}

1. Yang X, Yu Y, Xu J, Shu H, Xia J, Liu H, et al. Clinical course and outcomes of critically ill patients with SARS-CoV-2 pneumonia in Wuhan, China: a single-centered, retrospective, observational study. Lancet Respir Med. 2020;8:475-81. https://doi.org/10. 1016/s2213-2600(20)30079-5.

2. Huang C, Wang Y, Li X, Ren L, Zhao J, Hu Y, et al. Clinical features of patients infected with 2019 novel coronavirus in Wuhan, China. Lancet. 2020;395:497-506. https://doi.org/10. 1016/s0140-6736(20)30183-5.

3. White DB, Lo B. A framework for rationing ventilators and critical care beds during the COVID-19 pandemic. JAMA. 2020;323:1773. https://doi.org/10.1001/jama.2020.5046.

4. Zhou F, Yu T, Du R, Fan G, Liu Y, Liu Z, et al. Clinical course and risk factors for mortality of adult inpatients with COVID-19 in Wuhan, China: a retrospective cohort study. Lancet. 2020;395: 1054-62. https://doi.org/10.1016/s0140-6736(20)30566-3.
5. Wu C, Chen X, Cai Y, Xia J, Zhou X, Xu S, et al. Risk factors associated with acute respiratory distress syndrome and death in patients with coronavirus disease 2019 pneumonia in Wuhan, China. JAMA Intern Med. 2020;180:1-11. https://doi.org/10. 1001/jamainternmed.2020.0994.

6. Wu Z, McGoogan JM. Characteristics of and important lessons from the coronavirus disease 2019 (COVID-19) outbreak in China: summary of a report of 72314 cases from the Chinese Center for Disease Control and Prevention. JAMA. 2020;323: 1239. https://doi.org/10.1001/jama.2020.2648.

7. Simpson S, Kay FU, Abbara S, Bhalla S, Chung JH, Chung M, et al. Radiological Society of North America Expert Consensus statement on reporting chest CT findings related to COVID-19. Endorsed by the Society of Thoracic Radiology, the American College of Radiology, and RSNA. Radiol Cardiothorac Imaging. 2020;2:e200152. https://doi.org/10.1148/ryct.2020200152.

8. Bernheim A, Mei X, Huang M, Yang Y, Fayad ZA, Zhang N, et al. Chest CT findings in coronavirus disease-19 (COVID-19): relationship to duration of infection. Radiology. 2020;295:200463. https:// doi.org/10.1148/radiol.2020200463.

9. Wang Y, Dong C, Hu Y, Li C, Ren Q, Zhang X, et al. Temporal changes of CT findings in 90 patients with COVID-19 pneumonia: a longitudinal study. Radiology. 2020;296:E55-64. https://doi.org/ 10.1148/radiol.2020200843.

10. Salehi S, Abedi A, Balakrishnan S, Gholamrezanezhad A. Coronavirus disease 2019 (COVID-19): a systematic review of imaging findings in 919 patients. AJR Am J Roentgenol. 2020;215:87-93. https://doi.org/10.2214/ajr.20.23034.

11. Pan Y, Guan H, Zhou S, Wang Y, Li Q, Zhu T, et al. Initial CT findings and temporal changes in patients with the novel coronavirus pneumonia (2019-nCoV): a study of 63 patients in Wuhan, China. Eur Radiol. 2020;30:3306-9. https://doi.org/10.1007/ s00330-020-06731-x.

12. Ai T, Yang Z, Hou H, Zhan C, Chen C, Lv W, et al. Correlation of chest CT and RT-PCR testing in coronavirus disease 2019 (COVID-19) in China: a report of 1014 cases. Radiology. 2020;296:E32-40. https://doi.org/10.1148/radiol.2020200642.

13. Mahdavi A, Khalili N, Davarpanah AH, Faghihi T, Mahdavi A, Haseli S, et al. Radiologic management of COVID-19: preliminary experience of the Iranian Society of Radiology COVID-19 Consultant Group (ISRCC). Iran J Radiol. 2020;e102324. https:// doi.org/10.5812/iranjradiol.102324.

14. Davarpanah AH, Mahdavi A, Sabri A, Langroudi TF, Kahkouee S, Haseli S, et al. Novel screening and triage strategy in Iran during deadly coronavirus disease 2019 (COVID-19) epidemic: value of humanitarian teleconsultation service. J Am Coll Radiol. 2020;17: 734-8. https://doi.org/10.1016/j.jacr.2020.03.015.

15. Vice-Chancellor's Office in Treatment Affairs. Shahid Beheshti University of Medical Sciences T, Iran. Guidelines for COVID19. 2020. Accessed 17 April 2020. Available from: http:// treatment.sbmu.ac.ir/index.jsp?siteid $=62 \&$ fkeyid $=\&$ siteid $=$ 62\&pageid $=63989$

16. Koo HJ, Lim S, Choe J, Choi SH, Sung H, Do KH. Radiographic and CT features of viral pneumonia. Radiographics. 2018;38:719 39. https://doi.org/10.1148/rg.2018170048.

17. Zhou S, Wang Y, Zhu T, Xia L. CT features of coronavirus disease 2019 (COVID-19) pneumonia in 62 patients in Wuhan, China. AJR Am J Roentgenol. 2020;214:1287-94. https://doi.org/10.2214/ajr. 20.22975 .

18. Emanuel EJ, Persad G, Upshur R, Thome B, Parker M, Glickman A, et al. Fair allocation of scarce medical resources in the time of COVID-19. N Engl J Med. 2020;382:2049-55. https://doi.org/10. 1056/NEJMsb2005114.

19. Zhang J, Zhou L, Yang Y, Peng W, Wang W, Chen X. Therapeutic and triage strategies for 2019 novel coronavirus disease in fever 
clinics. Lancet Respir Med. 2020;8(3):e11-e2. https://doi.org/10. 1016/s2213-2600(20)30071-0.

20. Ayebare RR, Flick R, Okware S, Bodo B, Lamorde M. Adoption of COVID-19 triage strategies for low-income settings. Lancet Respir Med. 2020;8(4):e22. https://doi.org/10.1016/s2213-2600(20) 30114-4.

21. Xie J, Tong Z, Guan X, Du B, Qiu H, Slutsky AS. Critical care crisis and some recommendations during the COVID-19 epidemic in China. Intensive Care Med. 2020;46:837-40. https://doi.org/10. 1007/s00134-020-05979-7.

22. CDC COVID-19 Response Team. Severe outcomes among patients with coronavirus disease 2019 (COVID-19) - United States, February 12-March 16, 2020. MMWR Morb Mortal Wkly Rep. 2020;69(12):343-6. https://doi.org/10.15585/mmwr.mm6912e2.

23. Villar J, Pérez-Méndez L, Basaldúa S, Blanco J, Aguilar G, Toral $\mathrm{D}$, et al. A risk tertiles model for predicting mortality in patients with acute respiratory distress syndrome: age, plateau pressure, and $\mathrm{P}(\mathrm{aO}(2)) / \mathrm{F}(\mathrm{IO}(2))$ at ARDS onset can predict mortality. Respir Care. 2011;56(4):420-8. https://doi.org/10.4187/respcare.00811.

24. Pennica A, Conforti G, Falangone F, Martocchia A, Tafaro L, Sentimentale A, et al. Clinical management of adult coronavirus infection disease 2019 (COVID-19) positive in the setting of low and medium intensity of care: a short practical review. Sn Compr Clin Med. 2020;2:694-9. https://doi.org/10.1007/s42399-02000333-w.
25. Liu Y, Yan LM, Wan L, Xiang TX, Le A, Liu JM, et al. Viral dynamics in mild and severe cases of COVID-19. Lancet Infect Dis. 2020;20:656-7. https://doi.org/10.1016/s1473-3099(20) 30232-2.

26. Bewick T, Greenwood S, Lim WS. What is the role of pulse oximetry in the assessment of patients with community-acquired pneumonia in primary care? Prim Care Respir J. 2020;19(4):378-82. https://doi.org/10.4104/pcrj.2010.00049.

27. Caruso D, Zerunian M, Polici M, Pucciarelli F, Polidori T, Rucci C, et al. Chest CT features of COVID-19 in Rome, Italy. Radiology. 2020;296(2):E79-85. https://doi.org/10.1148/radiol.2020201237.

28. Li K, Wu J, Wu F, Guo D, Chen L, Fang Z, et al. The clinical and chest CT features associated with severe and critical COVID-19 pneumonia. Investig Radiol. 2020;55:327-31. https://doi.org/10. 1097/RLI.0000000000000672.

29. Rubin GD, Ryerson CJ, Haramati LB, Sverzellati N, Kanne JP, Raoof $\mathrm{S}$, et al. The role of chest imaging in patient management during the COVID-19 pandemic: a multinational consensus statement from the Fleischner Society. Radiology. 2020;296:172-80. https://doi.org/10.1148/radiol.2020201365.

Publisher's Note Springer Nature remains neutral with regard to jurisdictional claims in published maps and institutional affiliations. 